\title{
Equation-Based Dynamic Shaping Algorithm
}

\author{
Halima Elbiaze, Tijani Chahed, Gérard Hébuterne, and Tulin Atmaca \\ Institut National des Télécommunications \\ 9 rue Charles Fourier 91011 Evry CEDEX - France \\ \{halima.elbiaze, tijani.chahed, gerard.hebuterne, tulin.atmaca\}@int-evry.fr \\ Phone : +33160764795, Fax : +33160764780
}

\begin{abstract}
The focus of this paper is the traffic shaping at the access of an network node. We propose a novel algorithm that dynamically shapes the incoming traffic, based on service curves equations, in order to meet the QoS constraints in terms of buffer size or delay. We first estimate arrival parameters within various time intervals in order to make the incoming traffic fit into a token bucket traffic specification (Tspec) format. We then derive the shaping parameters based on deterministic service curves. Those shaping parameters vary dynamically according to the Tspec of every time window.
\end{abstract}

\section{Introduction}

The traffic shaping takes place in the network edges to maintain the logical performance to its highest level and thus respect the traffic contract. The problem studied in this paper 1 , is motivated by the desire to obtain applicable performance bounds for a very high-speed network. To achieve this aim, a tool for studying end-to-end, bounded performance is needed. Classical queuing analysis studies average performance for aggregate traffic. It focuses on single server environments for traffic with somehow periodic inter-arrival times. However, in the packet-switching, integrated services models, bounds on the end-to-end performance need to be studied in a networking environment with traffic dynamics, interactions and burstiness far more complex than in the previous case. In this work, we use the deterministic version of the service curves method [2] and particularly Network Calculus (NC) [1, its Min-Plus algebra formulation.

\section{End-to-End System}

For source modeling, we consider the Deterministic Bounding IntervalDependent (D-BIND) model, found in [4]. It uses a family of rate-interval pairs where the rate is a bounding rate over the corresponding interval length. The

\footnotetext{
${ }^{1}$ supported by the "Réseau National de Recherche en Télécommunications" under the decision number 99S 0201-0204 and the European IST Project DAVID (Data and Voice Integration over DWDM)
} 


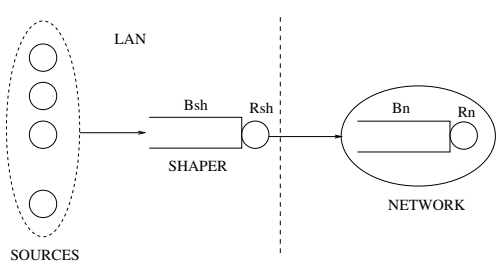

Fig. 1. End-to-end System

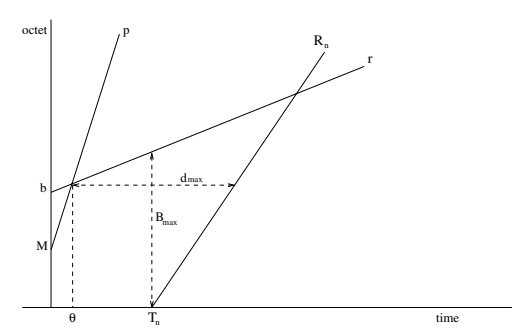

Fig. 2. Arrival Tspec and service curve

bounding rates used in this study are base on Traffic Specification (Tspec) formulation. Introduced by the IETF for IP, Tspec takes the form of a token bucket, with rate $r$ and depth $b$, plus a peak rate $p$ and maximum packet size $M$. We now show how sources traffic can be made to fit into a token bucket Tspec format. We partition the whole process into $N$ equal, non-overlapping blocks, corresponding to time intervals $\left(I_{i}\right)_{i=1, \ldots, N}$, of length $l_{N}$, and approximate the traffic volume within each interval $I_{i}$ by a corresponding set of Tspec parameters. In doing so, we obtain a piece-wise Tspec formulation of the global process which approximates the actual process.

Using the service curve approach, the arrival curve corresponding to the Tspec given in terms of $p, M, r, b$, equals $\min (p t+M, r t+b)$. The network is modeled by $n$ elements in tandem, each one with service rate $R_{i}$ and starting service at time $T_{i}, i=1, . ., n$, one possible concatenation scenario is a network element with a service curve $c(t)=R_{n}\left(t-T_{n}\right)^{+}$where $R_{n}=\min \left(R 1, R 2, . ., R_{n}\right)$ and $T_{n}=$ $\sum_{i}^{n} T_{i}$. Figure 2 represents an arrival Tspec and service curve, showing the corresponding fundamental bounds of Network Calculus [1, namely the backlog $B_{\max }$ and the delay $d_{\max }$.

\section{Shaping}

Adding a shaper to the arriving traffic prior to its entrance to the network is done as follows. A new service curve, corresponding to the actions of the shaper, with parameters $\left(R_{s h}, T_{s h}\right)$ is set between the arrival curve and the network service curve. In what follows, we assume, without loss of generality, $T_{n}=0$.

\subsection{Shaping to Meet Buffer Requirement and/or Delay Constraint}

Let us suppose that the maximum buffer size, $B_{c}$, at the network level is smaller than the maximum backlog bound, $B_{\max }$, caused by the non-shaped arriving traffic. The point of introducing a shaper in this case is to assure that the incoming traffic does not exceed $B_{c}$ for a loss-free network performance.

Schematically, and considering the setting of Figure 3 the idea is to vary the shaping curve through the segment indicating $B_{c}$. In this case, the shaded region, given in Figure 3, shows the region of shaping. Through Network Calculus 
[1] bounds formulations, we easily derive maximal $R_{s h}$ (buffer constraint) corresponding to optimal shaping case.

In delay constraint case, the point of shaping is to reduce the maximum delay to be experienced at the network region from the original $d_{\max }$ to a new delay constraint $d_{c}$. Let us note that introducing a shaper does not add to the end-toend delay. This is again achieved by setting appropriate values to $R_{s h}$ (delay constraint). Figure 4 shows the shaping region varying the line the shaping curve through the segment indicating $d_{c} . R_{s h}$ (buffer constraint $)=\frac{B_{c} r-R_{n} b}{B_{c}-b}(*)$ ; $\quad R_{s h}$ (delay constraint $)=\frac{R_{n}\left(b-r d_{c}\right)}{b-R_{n} d_{c}}(* *)$

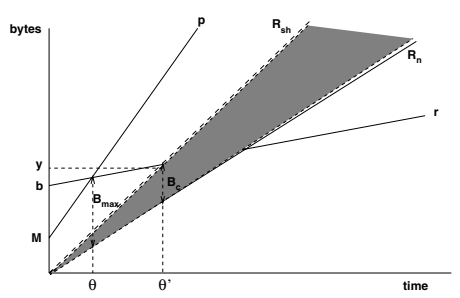

Fig. 3. Region of shaping to meet buffer requirement $B_{c}$

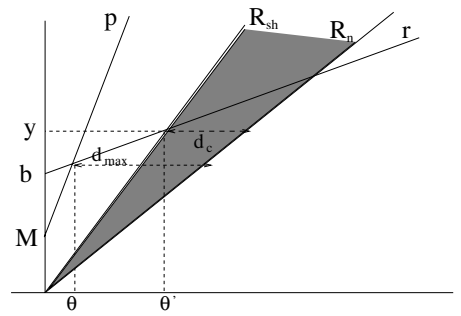

Fig. 4. Region of shaping to meet delay requirement $d_{c}$

\subsection{Equation-Based Dynamic Shaping Algorithm}

So far, we considered shaping within every interval $I_{i}$ of length $l_{N}$. Our ultimate aim is however to shape the global incoming traffic. Parallel to the idea of partitioning the arrival process so as to locally bound each interval, the shaping scheme introduced in the previous section shall apply to each interval.

It is clear that the shaping rate $R_{s h}$ depends on the arrival curve parameters throughout the whole process. The task in this case is to find optimal, i.e. maximal, shaping rate $R_{s h}$ for each interval $I_{i}$ such that the buffer constraint and/or delay constraint are satisfied. This is achieved by dynamically changing the shaping rate from one interval to the next. The dynamic shaping algorithm is then as follows.

1. Set observation window size equal to $l_{N}$

2. Determine corresponding Tspec in interval $\left(I_{i}\right)_{i=1, \ldots, N}$

3. Apply Equations $(*)$ and $(* *)$. to set shaping parameters such that i. shaping is optimall : minimal buffer size $B_{s h}$ and maximal shaping rate $R_{s h}$

ii. requirements are met, i.e., buffer or delay constraint at network level iii. no loss at shaper, i.e. $B_{s h}$ not exceeded. 


\section{Numerical Results}

The end-to-end system studied is shown in Figure 1. We consider self similar traffic resulting from the LAN sources with mean $=100 \mathrm{Mbit} / \mathrm{s}$, variance $=10^{9}$, Hurst parameter $\mathrm{H}=0.7$, and $\mathrm{M}$ equal to 1540 bytes. At the network level, let $R_{n}=227 \mathrm{Mbit} / \mathrm{s}$ be the rate of the server, with buffer capacity $B_{n}$ equal to 100 packets.

\subsection{Estimation of Arrival Parameters}

We first estimate the parameters of the incoming traffic into a Tspec format, for different observation windows of size $l_{N}$.

Figures 5 and 6 shows the average rate $r_{i}$ for intervals $I_{i}$ of lengths equal to $300 \mathrm{~ms}$ and $1 \mathrm{~s}$ respectively. We notice that for a given window size $l_{N}, r_{i}^{N}$ varies from one interval to the next keeping the same behavior as the original traffic, i.e., incorporating correlation. On the other hand, the family of $\left(r_{i}^{N}\right)_{i=1, \ldots, N}$ behaves
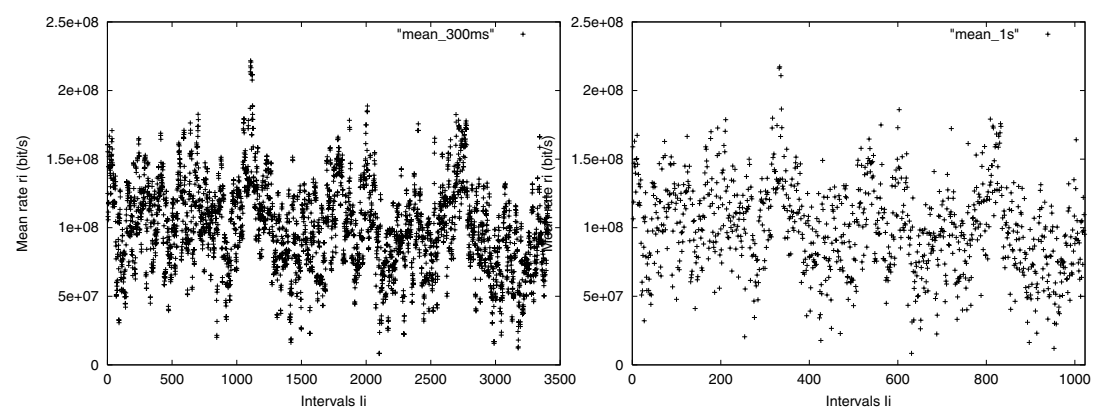

Fig. 5. Average rate $r_{i}$ for $l_{N}=300 \mathrm{~ms}$

Fig. 6. Average rate $r_{i}$ for $l_{N}=1 s$

in the same way in different lengths $l_{N}$ of intervals $\left(I_{i}\right)_{i=1, \ldots, N}$, i.e., in many time scales. That means the presence of self-similarity property in the sequence $\left(r_{i}^{N}\right)_{i=1, \ldots, N}$. Figures [5 and [6] confirms that for two times scales $\left(r_{i}^{N}\right)_{i=1, \ldots, N}$ behaviors : $300 \mathrm{~ms}$ and $1 \mathrm{~s}$. The same remarks remain valid for $\mathrm{p}$.

The burst size b estimation consists on the sum of consecutive interarrivals smaller or equal to the interarrival time within the corresponding peak rate $p_{i}$ corresponds to the burst size. The obtained values for $\mathrm{b}$ vary from 85 packets for small window size $l_{N}$ to 60 for larger ones.

\subsection{Performance}

If no shaping is used at the access of the network, a 55 packet buffer size is needed at the network server to achieve no-loss performance. The maximum delay in the network level in this case is equal to $0.00268 \mathrm{sec}$. 
Let us assume that in fact, the buffer size $B_{c}=10$ at the network level cannot be as large as to hold 55 packets, i.e., if no shaping is used, there will be some loss. We derive the shaping parameters for every interval $\left(I_{i}\right)_{i=1, \ldots, N}$ of length $l_{N}$. For intervals of length $l_{N}=100 \mathrm{~ms}$, Figure 7 shows the mean arrival rate $r_{i}$ versus shaping rate $R_{s h}$ throughout the duration of the connection (100 seconds). We
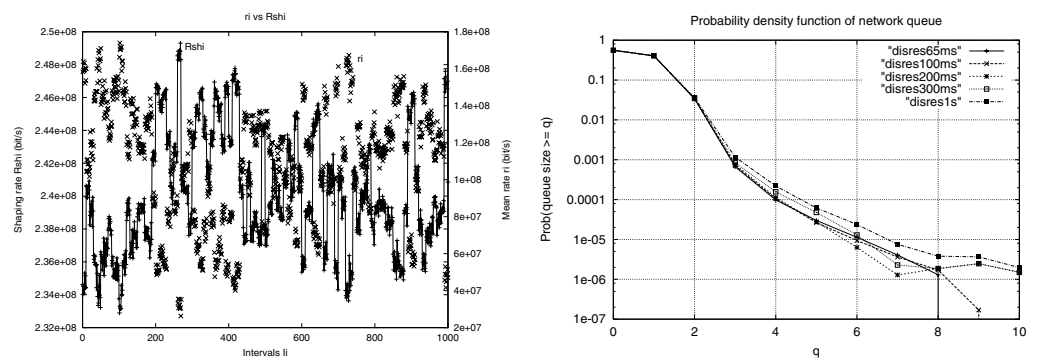

Fig. 7. Average rate $r_{i}$ vs Shaping rate Fig. 8. Network queue PDFs for Interval $R_{s h}$ during the Interval $I_{i}$ $I_{i}$ lengths: $65 \mathrm{~ms}, 100 \mathrm{~ms}, 200 \mathrm{~ms}, 300 \mathrm{~ms}, 1 \mathrm{~s}$

notice that $r_{i}$ and $R_{s h}$ are inversely proportional; for every large values of $r_{i}$, i.e., high input rate, the value of $R_{s h}$ is small, i.e., a severe shaping is needed to accommodate the buffer constraint and the loss-free performance. This done,we plug the equation-based shaping parameters back into the simulation model. No loss is observed at the shaper for different observation window lengths $l_{N}$ because we consider shaper size is largest value over all intervals derived from the equations. This independence between the shaping queue PDF's and the interval sizes $l_{N} \mathrm{~s}$, can be explained by the fact that the shaping rate $R_{s h}$ is adaptive with respect to the incoming traffic in order to meet the non-loss performance. Figure 8 shows the probability density function of the buffer occupancy at the network level for different observation window lengths $l_{N}$. We notice that the smallest interval lengths $l_{N}=65$ and $100 \mathrm{~ms}$ yield a non-loss performance. This is explained by the fact that at those interval lengths, we obtain higher precision for estimation of arrival parameters and hence shaping parameters. For larger interval lengths, $l_{N}=200$ and 300 and $1000 \mathrm{~ms}$, some loss, on the order of 2.4 $10^{-7}$, is observed. This is explained by the fact that for small precision, the arrival parameters are under-estimated. Put in the equations, they yield high shaping rates, or equivalently, soft shaping. This in turn results in loss at the network level. We follow the same steps as buffer-constrained performance to respect the tolerated maximum delay $d_{c}=0.0005$ at the network level.

Table1illustrates the maximum delay values obtained by simulation for different window lengths $l_{N}$ : $65 \mathrm{~ms}, 100 \mathrm{~ms}, 200 \mathrm{~ms}, 300 \mathrm{~ms}$ and $1 \mathrm{~s}$. Again, we notice that the smallest interval lengths $l_{N}=65$ and $100 \mathrm{~ms}$ yield the target maximum delay.

For larger interval lengths, $l_{N}=200$ and 300 and $1000 \mathrm{~ms}$, the maximum values 
of the observed delay exceed the constraint. This is explained by the fact that for small precision, the arrival parameters are under-estimated.

Table 1. Maximum delay at the network level for different window lengths $l_{N}: 65 \mathrm{~ms}$, $100 \mathrm{~ms}, 200 \mathrm{~ms}, 300 \mathrm{~ms}, 1 \mathrm{~s}$

\begin{tabular}{|c|c|c|c|c|c|}
\hline window lengths $l_{N}$ & $\mathbf{6 5 m s}$ & $\mathbf{1 0 0 m s}$ & $\mathbf{2 0 0 m s}$ & $\mathbf{3 0 0 m s}$ & 1s \\
\hline maximum delay a the network & $0.0004102 \mathrm{~s}$ & $0.0004219 \mathrm{~s}$ & $0.0005413 \mathrm{~s}$ & $0.0005414 \mathrm{~s}$ & $0.0005414 \mathrm{~s}$ \\
\hline
\end{tabular}

\section{Conclusion}

In this paper, we focused on self-similar traffic [5] at the input of an network node. If this traffic is left as is, it cannot satisfy the buffer and/or delay constraint at the network level, which may be very stringent in the optical case. In order to meet those requirements, shaping is essential. In this work, we proposed an equation-based dynamic shaping with three key steps: 1) estimation and fitting of interval-wise incoming traffic into arrival curves, 2) solving into the service curve approach for the shaping parameters in an adaptive manner and 3) fitting the later back into the original model.

As of the first step of our algorithm, we notice that the input estimate reproduce the same self-similar, correlated nature of the original traffic. The shaping parameters derived in step 2 are typically conservative owing to the deterministic nature of the service curve. However, when put back into the original model, i.e., step 3, they are shown to be numerically not very conservative. This may be explained by the correlated nature of the original self-similar traffic.

\section{References}

1. J.-Y. Le Boudec Application of Network Calculus To Guaranteed Service Networks. IEEE Trans on Information theory, (44) 3, May 1998.

2. R. L. Cruz Quality of Service Guarantees in Virtual Circuit Switched Networks. IEEE JSAC, 1995.

3. C. Kamanek, H. Kanakia and S. Keshav. Rate controlled servers for very highspeed networks In Proceedings of IEEE Global Telecommunications Conference, pp. 300.3.1-300.3.9,San Diego, California, December 1990.

4. E. Knightly and H. Zhang. Traffic characterization and switch utilization usinf deterministic bounding interval dependent traffic models In Proceedings of IEEE INFOCOM'95,Boston, MA, April 1995.

5. V. Paxon and S. Floyd. Wide-Area Traffic: The failure of Poisson Modeling IEEE/ACM Transactions on Networking, 3(3), pp; 226-244, June 1995. 